\title{
Research on Buffer Capacity Allocation of Engine Cylinder Block Production Line Based on SA - PSO Algorithm
}

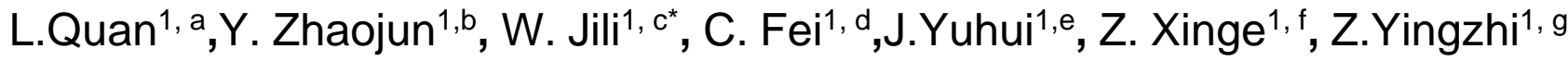 \\ ${ }^{1}$ College of Mechanical Science and Engineering, Jilin University, Changchun 130022, China \\ ajlu_liquan@163.com, byangzj@126.com, 'wangjili1100@126.com, dchenfeicn@jlu.edu.cn, \\ e jiayuhui@163.com, 'zhangxinge@jlu.edu.cn, grhangyz@jlu.edu.cn
}

\begin{abstract}
Keywords: Production line, Buffer capacity allocation, Availability model ,SA - PSO algorithm
Abstract. In order to allocate the buffer capacity effectively and improve the availability of the production line, a buffer allocation method based on particle swarm optimization (PSO) and simulated annealing algorithm (SA) is proposed for the mixed asynchronous serial production line. It is considered that the production line includes series equipment and parallel equipment and the processing capacity of the equipment is not the same. On the basis of ensuring that the performance of the production line does not change obviously, the production line is transformed into a synchronous production line, and the availability model of the production line is established by analytic method. The SA algorithm is used to improve the PSO. The performance of the algorithm is validated and analyzed. At the same time, a concrete example is given to show that the buffer allocation method is feasible and effective.
\end{abstract}

\section{Introduction}

Nowadays enterprises pay more attention to research the performance and reliability of the production line, the buffer can effectively improve the performance of the production line and alleviate the loss caused by the failure of a single device in the system, so a reasonable buffer allocation method is of great significance.

The optimization design of buffer capacity is mainly to meet performance index and reliability index of the production line. Zhou B H [1] established the availability evaluation model of the serial production line and the buffer capacity was optimized by genetic algorithm. The equipment priority method was adopted for the serial production line by Papadopoulos H T [2], heuristic search algorithm was used for the distribution of the buffer capacity, The algorithm can improve the speed of solution effectively. Demir L [3] used the decomposing method to obtain the throughput evaluation model for the asynchronous production line. An adaptive tabu search algorithm was used to determine the buffer capacity of the production line, but the algorithm was easy to fall into the local optimum. The same decomposition method was used to establish throughput evaluation model by LEYUAN SHI [4], combined with hybrid nested partition and tabu search algorithm for the allocation of buffer capacity, which greatly improves the search efficiency. Pan X [5] analyzed the performance evaluation model of the hybrid production line by the decomposition method and queuing theory, and used the simulated annealing algorithm to allocate the buffer capacity, and considered the cost factor. Meerkov S M [6] used the merger method to obtain the efficiency evaluation model of production line and combined with the iterative search algorithm on the buffer capacity allocation. Gershwin [7] used the decomposition method to get the throughput performance evaluation model, and used a gradient algorithm to allocate the buffer capacity of the serial production line efficiently, but it was difficult to deal with the problem of complicated production lines. The above studies aim to maximize the availability and throughput of the production line or to minimize the inventory level and cost, the relevant optimization algorithms are used to optimize the buffer capacity, but most of them are for simple synchronous serial production lines.

In this paper, a hybrid algorithm of particle swarm optimization (PSO) and simulated annealing algorithm (SA) is used to optimize the buffer capacity of mixed asynchronous production lines. The performance of the algorithm is analyzed and compared with other algorithms in the concrete examples. 


\section{Availability model of production line}

In this paper, we study a repairable hybrid asynchronous serial production line with buffers, which is converted into a simple serial production line by means of KUT C [8]. At the same time, the processing capacity of each machine is not the same in the production line, so first it is necessary to convert the asynchronous production line into the synchronous production line, and according to the relationship between the availability of synchronous production line and the buffer capacity, the availability model is established.

In order to simplify the analysis process and reflect the real dynamic system, a flow model method is proposed to solve the problem. According to Lim, J.-T [9]'s Production line assumptions, converting the asynchronous production line to the synchronous production line is divided into the following steps:

- The processing capacity $C_{i}$ of each machine is different and throughput is used as the performance index in the system. The processing capacity indicates the number of pieces processed per unit time, the failure rate and the maintenance rate are respectively $\lambda_{i}$ and $\mu_{i}$.The throughput $T P_{i}$ of each machine in the system is:

$$
T P_{i}=C_{i} \frac{\mu_{i}}{\lambda_{i}+\mu_{i}}
$$

By using the Jacobs D A iterative merging method [10], we can calculate the throughput value $T P_{s}$ of asynchronous production line.

- To ensure that throughput $T P_{i}$ of the machine in the production line remained unchanged, the failure rate maintains the original reliability level and the maintenance rate is adjusted, so that asynchronous production lines can be transformed into synchronous production lines, from the Formula (1) to obtain the maintenance rate of each machine:

$$
\mu_{i}=\frac{T P_{i} \lambda_{i}}{C_{i}-T P_{i}}
$$

After the transformation, the processing capacity of the machine in the synchronous production line is $C_{s}$, it needs to meet the following conditions:

$$
\max _{i=M} T P_{i}<C_{s}<\max _{i=M} C_{i}
$$

In the interval of $\left(\max _{i=M} T P_{i}, \max _{i=M} C_{i}\right)$, the one-dimensional ergodic search method is used. Let the search precision be $\Delta$.By using the formula (2), the maintenance rate $\mu_{i}$ can be obtained to meet this processing capacity $C_{s}$ of the synchronization line. According to Jacobs DA's iterative merging method, we can calculate the throughput $T P_{s}$ of synchronous production line.

- The throughput relative error is $\Delta T P=\left|T P_{s}-T P_{a}\right|$, we can search the smallest relative error $\min (\Delta T P)$ by the one-dimensional ergodic search method, in this case the processing capacity value $C_{0}$ is the optimal value in the synchronous production line.

After transforming to synchronous production line, the processing capacity of each machine in the production line is identical. According to Malathronas[11]'s availability model of two-machine production line and Sörensen K[12]'s iterative merging method, The availability $A_{e s}$ of the synchronous serial production line is:

$$
\begin{aligned}
& A_{e s}=A_{e(n-1)}=\frac{\left[\rho_{e(n-2)}-\rho_{n} \exp \left(-k_{n-2}\right)\right] A_{e(n-2)} A_{n}}{\rho_{e(n-2)} A_{n}-\rho_{n} A_{e(n-2)} \exp \left(-k_{n-2}\right)} \\
& k_{n-2}=\frac{\left(\mu_{e(n-2)}+\mu_{n}+\lambda_{e(n-2)}+\lambda_{n}\right)\left(\lambda_{e(n-2)} \mu_{n}-\lambda_{n} \mu_{e(n-2)}\right)}{\left(\mu_{e(n-2)}+\mu_{n}\right)\left(\lambda_{e(n-2)}+\lambda_{3}\right) k_{n-1}}
\end{aligned}
$$

The buffer size is $V_{i}, k_{n-1}$ represents the ratio of $C_{0}$ and $V_{n-1}$, which $C_{0}$ is the optimal value of processing capacity in the synchronous production line. $A_{e(n-2)}$ is the availability of the machine $M_{e(n-2)}, \rho_{e(n-2)}$ is the ratio of failure rate $\lambda_{i}$ and maintenance rate $\mu_{i}, \mu_{e(n-2)}$ is maintenance rate of $M_{e(n-2)}, \lambda_{i}$ is failure rate of $M_{e(n-2)} \cdot M_{e(n-2)}$ is obtained by the iterative merging method . 


\section{Buffer capacity allocation based on SA - PSO algorithm}

There are two main types of buffer capacity optimization problems. One is to minimize the cost of the production line (buffer capacity) and the other is maximize the output of the production line. In this paper, the buffer capacity is constrained to maximize the output of the production line, so the buffer capacity optimization model is:

$$
\begin{aligned}
& \text { Max } A_{\text {es }}\left(V_{1}, V_{2}, \cdots V_{n-1}\right) \\
& \text { s.t. }\left\{\begin{array}{l}
\sum_{i=1}^{n} V_{i} \leq m \\
V_{i} \geq 1
\end{array}\right.
\end{aligned}
$$

In order to obtain the optimal buffer allocation, firstly the constrained optimization model is transformed into unconstrained optimization model, and then the SA-PSO algorithm is used to solve the buffer capacity optimization model. Since the external penalty function method does not need to provide the initial feasible solution, this paper uses the external penalty function method, the solution is moving from the non-feasible region to the feasible region. According to the penalty function method, the availability model of production line is transformed into the unconstrained optimization model:

$$
\operatorname{Min} F(x)=-A\left(V_{1}, V_{2}, \cdots V_{n-1}\right)+\sigma \sum_{i=1}^{m}\left[\max \left\{0, \sum_{i=1}^{M-1} V_{i}-m\right\}\right]^{2}+\sigma \sum_{i=1}^{m}\left[\max \left\{0,1-V_{i}\right\}\right]^{2}
$$

In this paper, the unconstrained optimization model of production line is taken as the fitness function. The improved particle swarm optimization algorithm has been widely used to balance the production line [13] [14]. Compared with genetic algorithm, particle swarm optimization (PSO) has the advantages of real number coding and fast speed of computation, but it is easy to fall into local optimum for complex nonlinear problems. In this paper, particle swarm optimization (PSO) algorithm based on simulated annealing (SA) is proposed. PSO has constraint factor [15], which can ensure the convergence of PSO algorithm and eliminate the limitation of velocity boundary by selecting appropriate parameters. In order to avoid falling into the local minimum solution, a suboptimal solution is chosen to replace the global optimal solution $P_{g}$ in the speed updating formula. The mechanism of simulated annealing algorithm (SA) is used to guarantee the local optimal solution $P_{i}$ with a higher probability of being selected. When the temperature is T, the probability of being selected can be calculated by $e^{-\left(f_{p t}-f_{p s}\right) / t}$, Which $f$ represents the objective function value. If the probability value of being selected is taken as the adaptation value of the local optimal solution $P_{i}$, then the probability of $P_{i}$ instead of $P_{g}$ is:

$$
e^{-\left(f_{p t}-f_{p g}\right) / t} / \sum_{j=1}^{N} e^{-\left(f_{p t}-f_{p g}\right) / t}
$$

By using roulette strategy, we select one $P_{i}$ to take place of the global optimal solution $P_{g}$.

Based on the experimental design proposed by Gershwin [7], the SA-PSO algorithm is used to optimize the allocation of the buffer capacity of production line and compared with the genetic algorithm proposed by Zhang Liangan [16] and the PSO with adaptive weights and SA algorithms, the performance index is evaluated by relative error of availability. When the number of machines is 7 , the MTTR and MTBF values of each machine are shown in table 1 . The availability model of the production line is established, and the distribution result is optimized by the algorithm (Table 2). The iterative convergence process is shown in figure 1.

Table 1 The parameters of the machine

\begin{tabular}{llllllll}
\hline Machine & 1 & 2 & 3 & 4 & 5 & 6 & 7 \\
\hline MTTR/h & 450 & 760 & 460 & 270 & 270 & 650 & 320 \\
MTBF/h & 820 & 5700 & 870 & 830 & 970 & 1900 & 1100 \\
\hline
\end{tabular}


Table 2 The results of the buffer allocation

\begin{tabular}{lllllllllll}
\hline \multirow{2}{*}{ Case } & 1 & 2 & 3 & 4 & 5 & 6 & $N^{\text {total }}$ & $A_{\text {opt }}$ & \multirow{2}{*}{$A_{\text {est }}$} & \multirow{2}{*}{$\Delta / 10^{-5}$} \\
\hline SA-PSO & 1 & 1 & 1 & 18 & 1 & 32 & 54 & 0.296983 & 0.296966 & 5.72 \\
GA & 1 & 1 & 2 & 19 & 2 & 29 & 54 & 0.296983 & 0.296898 & 28.62 \\
AWPSO & 1 & 13 & 9 & 11 & 6 & 14 & 54 & 0.296983 & 0.296049 & 314.5 \\
SA & 1 & 4 & 2 & 7 & 2 & 38 & 54 & 0.296983 & 0.296873 & 37.04 \\
\hline
\end{tabular}

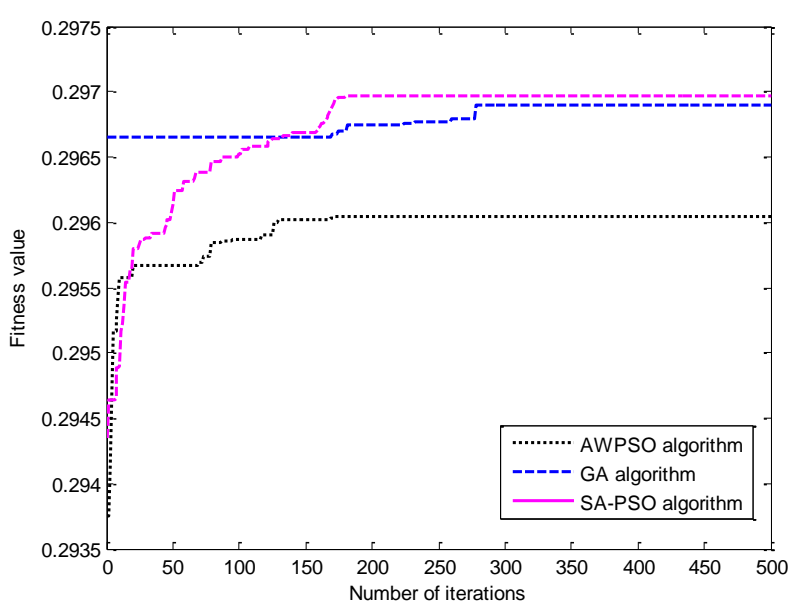

Figure 1 The comparison of algorithm performance

Figure (1) is the iterative result when the number of machines in the synchronous production line is 7. Compared with other algorithms, the SA-PSO algorithm has better convergence, better searching ability and higher precision of solution. According to table 2, from the optimization results of buffer capacity, the relative error of the availability is low by using the SA-PSO algorithm, so it is concluded that SA-PSO algorithm has some advantages in solving the problem of buffer capacity optimization.

\section{Application of engine cylinder block production line}

Figure 2 shows the layout of an engine cylinder block production line for a certain type of engine. The production line is divided into a rough machining part and a finish machining part. The rough machining part contains 20 machining centers and every parallel machining centers constitutes a work unit and two machining centers in the same work unit are processing the same product, each working unit is connected in series. The finish machining part contains three machining centers and they are connected in series, the production line also includes cleaning machines, airtight test machines, assembly machines, honing machines and online detection machines, there are mechanical arms and logistics buffers between series machines. These machines correspond to the different processing operation and follow the principle of processing operations.

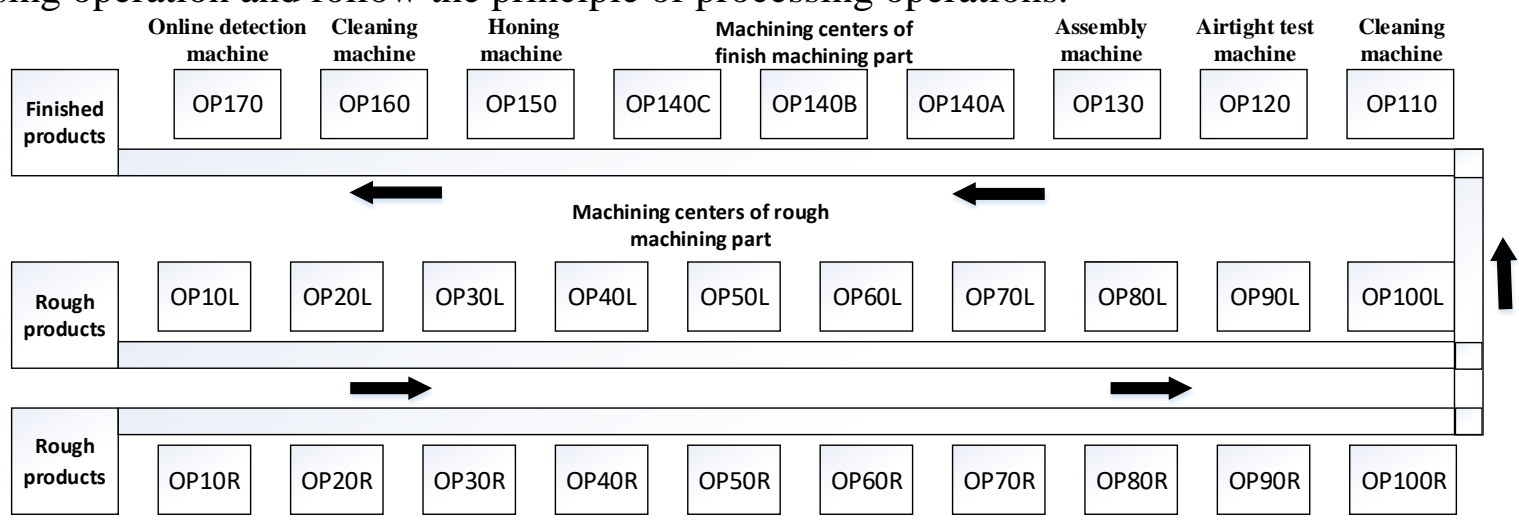

Figure 2 The layout of engine cylinder block production line 
The machines OP10-100R / L of the production line are respectively parallel connection, every parallel machines is equivalent to a work unit. At the same time, the machines OP140A / B / C of the production line are serial connection, these machines are also equivalent to a work unit, it can be seen from Figure 3 that there is a buffer between every two work units in the production line. To meet the continuity of production line, the first machine in the production line is not hungry and the last machine in the production line is not blocked.

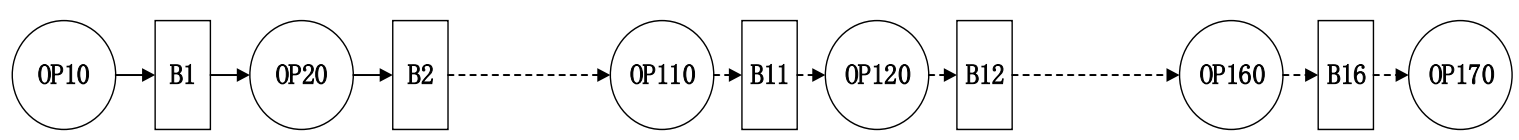

Figure 3 The simplified model of engine cylinder block production

As the production rate of the machines of the production line are different, the asynchronous production line needs to be transformed into the synchronous production line. The throughput $T P_{a}$ of the asynchronous line is calculated by using the method proposed in Section 1.1, the throughput $T P_{i}$ of each machine is calculated by using the formula (1). The one-dimensional Ergodic earch method is adopted, and the throughput error $\Delta$ is 0.05 . The searching precision of the processing ability value is 0.001 . The optimal processing capacity value $C_{0}$ of the synchronous production line is found to meet the requirement.

As the buffer capacity of the production line is limited by the floor space of the plant, it is necessary to increase the availability of the production line under the limited area. The two restrictions for buffer capacity are as follows:

$$
\begin{gathered}
\sum_{i=1}^{16} B_{i} \leq 160 \\
B_{i} \geq 1 \quad, i=1, \cdots, 16
\end{gathered}
$$

The availability model of the production line is proposed above, which is optimized by using the SA-PSO algorithm described in this paper. The unconstrained optimization model of cylinder production line is established by using the external penalty function method. The SA-PSO algorithm is used to solve the optimization model.

When the sample size is respectively 100 and 200, the fitness function value of the SA-PSO algorithm are shown in Fig. 4 and Fig.5. From the figure, it can be seen that the fitness function of the SA-PSO algorithm rises relatively quickly at first, then the results tend to be stable. It is shown that the SA-PSO algorithm can solve the problem quickly and it tends to the global optimal solution with the increasing of iteration number and the optimal solution can be obtained whether the sample size is large or small.

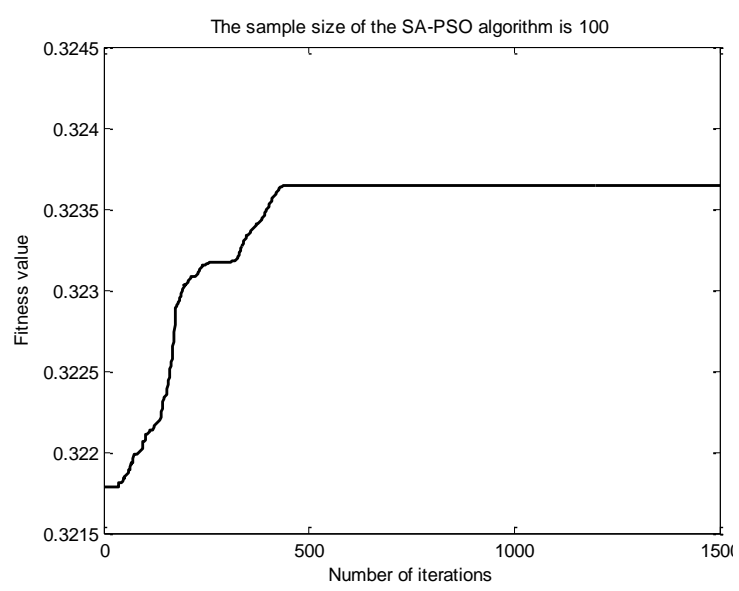

Figure 4 Iteration results with a sample size of 100

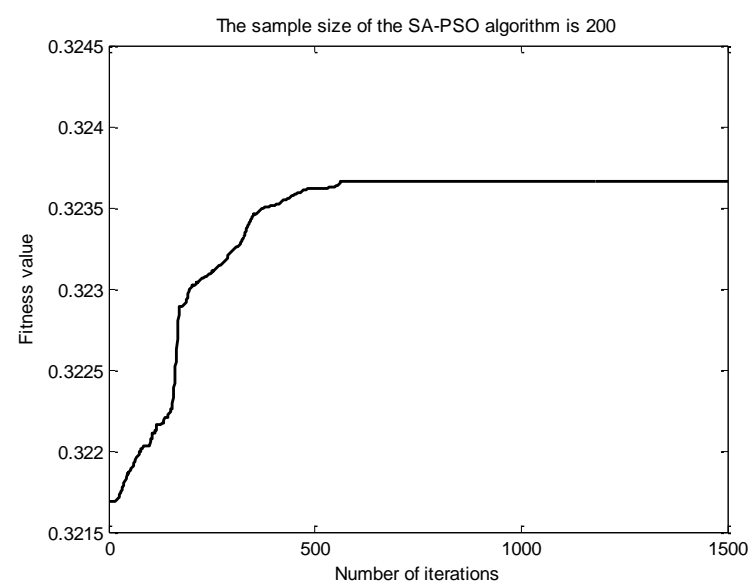

Figure 5 Iteration results with a sample size of 200 
The results of the SA-PSO algorithm for the optimization of the buffer capacity of the production line are shown in table 3.

Table 3 The results of buffer allocation of engine cylinder block production line

\begin{tabular}{|c|c|c|c|c|c|c|c|c|c|c|c|c|c|c|c|c|c|c|c|c|}
\hline \multirow{2}{*}{$\begin{array}{c}\text { Sample } \\
\text { size }\end{array}$} & \multicolumn{16}{|c|}{ Buffer i } & \multirow{2}{*}{$N_{\text {total }}$} & \multirow{2}{*}{$A_{o p t}$} & \multirow{2}{*}{$A_{\text {simu }}$} & \multirow[b]{2}{*}{$\Delta$} \\
\hline & 1 & 2 & 3 & 4 & 5 & 6 & 7 & 8 & 9 & 10 & 11 & 12 & 13 & 14 & 15 & 16 & & & & \\
\hline \multirow{3}{*}{100} & 1 & 1 & 4 & 9 & 9 & 12 & 1 & 1 & 9 & 28 & 41 & 1 & 32 & 1 & 4 & 6 & 160 & 0.322000 & 0.358537 & 0.036537 \\
\hline & 7 & 5 & 14 & 8 & 8 & 22 & 1 & 6 & 8 & 26 & 13 & 1 & 19 & 1 & 17 & 4 & 160 & 0.322139 & 0.374041 & 0.051902 \\
\hline & 12 & 1 & 17 & 2 & 10 & 11 & 4 & 7 & 22 & 20 & 10 & 1 & 21 & 1 & 13 & 8 & 160 & 0.322424 & 0.358445 & 0.036021 \\
\hline \multirow{5}{*}{200} & 13 & 1 & 17 & 4 & 10 & 8 & 4 & 7 & 22 & 19 & 11 & 1 & 21 & 1 & 13 & 8 & 160 & 0.322501 & 0.386467 & 0.063966 \\
\hline & 2 & 1 & 6 & 9 & 10 & 13 & 1 & 1 & 9 & 26 & 38 & 1 & 31 & 1 & 5 & 6 & 160 & 0.322253 & 0.378301 & 0.056048 \\
\hline & 5 & 3 & 16 & 9 & 9 & 22 & 1 & 5 & 7 & 28 & 13 & 1 & 19 & 1 & 17 & 4 & 160 & 0.322387 & 0.351845 & 0.029458 \\
\hline & 11 & 3 & 16 & 4 & 9 & 19 & 4 & 7 & 8 & 23 & 12 & 1 & 22 & 1 & 13 & 7 & 160 & 0.322207 & 0.347392 & 0.025185 \\
\hline & 12 & 1 & 15 & 6 & 8 & 15 & 4 & 5 & 9 & 26 & 15 & 1 & 22 & 1 & 13 & 7 & 160 & 0.322651 & 0.367577 & 0.044926 \\
\hline
\end{tabular}

From table 3, we know that when the sample size is respectively 100 and 200, four sets of optimal solutions are generated. The EM-plant simulation software is used to simulate and analyze the production line, The simulating time is $10^{5}$ unit time (s), Asimu is the average value of 100 simulation results.

Through the calculation of relative error $\Delta$, there is a certain error between the simulation result by EM-plant simulation software and the estimated result by using the SA-PSO algorithm, but The relative error is not big, which indicates that the avability model can be used to evaluate the production line by analytic method.

\section{Conclusions}

In this paper, we focus on the mixed asynchronous production line, the structural modeling method is used to simplify the analysis. On the basis of ensuring that the performance of the production line does not change obviously, the production line is transformed into a synchronous production line, and the availability model of the production line is established by analytic method. The optimal allocation of the buffer capacity of the production line is achieved by using the penalty function method and the SA-PSO algorithm with the objective of maximizing the availability of the production line.

The mechanism of simulated annealing algorithm (SA) is used to improve the global search performance of PSO algorithm. Compared with PSO algorithm with adaptive weights, genetic algorithm and SA algorithm, it is proved that the SA-PSO algorithm has obvious advantages, and its convergence speed is faster and it can avoid local optimization effectively.

This method is applied to the engine cylinder block production line, and the SA-PSO algorithm is used to solve availability model of the production line. According to the comparison, the buffer capacity optimization method proposed in this paper is feasible and effective .

\section{Acknowledgement}

This work is supported by the Important National Science and Technology Specific Projects of China(No. 2015ZX04003002 ) and Graduate Innovation Fund of Jilin University (No.2016179).

\section{References}

[1]. Zhou B H, Yu J D, Shao J Y. Buffer allocation method for series production lines based on availability assessments[J]. Zhejiang Daxue Xuebao (Gongxue Ban)/Journal of Zhejiang University (Engineering Science Edition, 2015, 49(5):893-900.

[2]. Papadopoulos H T, Vidalis M I. A heuristic algorithm for the buffer allocation in unreliable unbalanced production lines[J]. Computers \& Industrial Engineering, 2001, 41(3):261-277.

[3]. Demir L, Tunalı S, Eliiyi D T. An adaptive tabu search approach for buffer allocation problem in unreliable non-homogenous production lines[J]. Computers \& Operations Research, 2012, 39(7):1477-1486. 
[4]. LEYUAN SHI, SHULI MEN. Optimal buffer allocation in production lines[J]. IIE Transactions, 2003, 35(1):1-10.

[5].Pan X, Wu J, Zhang Q, et al. The Study of Buffer Allocation Problem in Complex Production Line[J]. Telkomnika Indonesian Journal of Electrical Engineering, 2014, 12(3).

[6].Hu A B, Meerkov S M. Lean buffering in serial production lines with Bernoulli machines[J]. Mathematical Problems in Engineering, 2006, 13(1):39-62.

[7].Gershwin SB, Schor JE. Efficient algorithms for buffer space allocation[J]. Annals of Operations Research, 2000, 93(1-4):117-144.

[8].KUT C. SO. Optimal buffer allocation strategy for minimizing work-in-process inventory in unpaced production lines[J]. Iie Transactions, 1997, 29(1):81-88.

[9]. Lim, J.-T, Meerkov, et al. Homogeneous, asymptotically reliable serial production lines: theory and case study[J]. IEEE Transactions on Automatic Control, 1990, 35(5):524-534.

[10].Jacobs D A. Improvability in production systems: Theory and case studies.[J]. 1993.

[11].John P. Malathronas, John D. Perkins, Richard L. Smith. The Availability of a System of Two Unreliable Machines Connected by an Intermediate Storage Tank[J]. Iie Transactions, 1983, 15(3):195-201.

[12].Sörensen K, Janssens G K. Buffer allocation and required availability in a transfer line with unreliable machines[J]. International Journal of Production Economics, 2001, 74(1-3):163-173.

[13].Xu L, Xu C, Wei D, et al. Machining Line Balancing of Engine Cylinder Block Based on SA-PSO Algorithm[J]. Nongye Jixie Xuebao/transactions of the Chinese Society of Agricultural Machinery, 2014, 45(2):16-21.

[14]. Wang Y, Feng Y, Tan J, et al. Optimization method of flexible job-shop scheduling based on multiobjective particle swarm optimization algorithm[J]. Transactions of the Chinese Society for Agricultural Machinery, 2011, 42(2):190-196.

[15]. Clerc M. The swarm and the queen: towards a deterministic and adaptive particle swarm optimization[C]//Evolutionary Computation, 1999. CEC 99. Proceedings of the 1999 Congress on. 1999.

[16]. Zhang L. Genetic Algorithm-based Buffer Allocation of a Robot Production Line[J]. Journal of Mechanical Engineering, 2010, 46(11):101-106. 